\title{
The Comparison of Size-Frequency Distributions of Impact Craters and Asteroids and the Planetary Cratering Rate
}

\author{
B. A. Ivanov \\ Institute for Dynamics of Geospheres, Russian Academy of Sciences \\ G. Neukum \\ DLR Institute of Space Sensor Technology and Planetary Exploration and \\ Institute of Geosciences, Free University of Berlin \\ W. F. Bottke Jr. \\ Southwest Research Institute \\ W. K. Hartmann \\ Planetary Science Institute
}

\begin{abstract}
The well-investigated size-frequency distributions (SFD) for lunar craters can be used to estimate the SFD for projectiles that formed craters both on terrestrial planets and on asteroids. Our results suggest these distributions may have been relative stable over the past 4 G.y. The derived projectile SFD is found to have a shape that is similar to the SFD of main-belt asteroids as compared with the astronomical observations (Spacewatch asteroid data, Palomar-Leiden survey, IRAS data) and in situ images obtained by space missions. This result suggests that asteroids (or, more generally, collisionally evolved bodies) are the main component of the family of impactors striking the terrestrial planets.
\end{abstract}

\section{INTRODUCTION}

Asteroids leaving the main belt (MB) may strike the Sun, be ejected out of the solar system, or create an impact crater when striking a terrestrial planetary body (i.e., planets, their satellites, or smaller bodies like asteroids or comets). Impacts craters have been measured and counted on all terrestrial planets and several asteroids. The relative age of different surfaces can be estimated by calculating crater densities, or the number of craters of a given diameter per unit area. Thus, crater densities provide planetologists with an instrument to compare the geological ages of various planetary surfaces, provided that the impactor flux striking each surface is known and the relationship between impact energy and crater size is well understood. All things being equal, larger crater densities imply older surfaces.

These issues have been recently covered by Neukum et al. (2001), Ivanov (2001), and Hartmann and Neukum (2001). In addition to crater chronology, the size-frequency distribution (SFD) of impact craters may be used to estimate the "production SFD" (often named "production function") of projectiles that formed those craters. To determine the production SFD, one must assume that that surface of the body was once a blank slate, such that the craters observed today directly reflect the size spectrum of the projectiles. Once we obtain a crater-derived "projectile" SFD, we can compare it to the SFD of observed asteroids and comets. In this way, we obtain some sense of how small bodies have evolved over time. On some surfaces, this integrated history may stretch all the way back to the "dark era" of the heavy bombardment period.

The projectiles capable of forming craters on the terrestrial planets today come primarily from three populations: (1) asteroids derived from the main belt, (2) Jupiter-family comets (JFCs) derived from the Kuiper Belt, and (3) long period comets (LPC) derived from the Oort Cloud (Morbidelli et al., 2002; Weissman et al., 2002). Other less-important contributors include the Trojan asteroids and the Halleytype comets.

Each impactor population mentioned above has undergone a specific, and possibly unique, accretion/collisional/ evolutionary history, such that their SFD are potentially quite different from one another. Moreover, these populations produce planet-crossing projectiles with characteristic orbits and physical properties, such that sorting out the importance of various impactor populations can be complicated. Fortunately, dynamical models and observational work provide some constraints. For example, Bottke et al. (2002a) show that asteroids, rather than JFCs or Trojans, currently provide most of the terrestrial planet impact craters coming from $a<7.4-\mathrm{AU}$ orbits (i.e., over 90\%). The fraction of craters formed by LPCs (active and dormant), however, is not well understood (see Weissman et al., 2002). To keep things as simple as possible, we will assume in this 
chapter that asteroids are the dominant source of impactors on the terrestrial planets.

The largest reservoir of asteroids in the inner solar system is the asteroid belt. As described in Bottke et al. (2002b) and Morbidelli et al. (2002), collisions, the Yarkovsky effect, and numerous mean-motion and secular resonances gradually push $\mathrm{D}<20-\mathrm{km}$ asteroids out of the main belt and onto planet-crossing orbits. We call this subpopulation the planet-crossing asteroids, or PCAs. PCAs are short-lived compared to the age of the solar system, such that new main-belt bodies must steadily resupply them over time. It is plausible that the differences between the main-belt SFD and that of the PCA populations are a consequence of the mechanisms producing new PCAs. Hence, a comparison between the SFD of terrestrial planet craters and those of various asteroid populations (e.g., main-belt asteroids, PCAs) should help find new constraints for future investigations of solar system evolution.

\section{LUNAR PRODUCTION FUNCTION}

The Moon is an ideal test site to study cratering records, particularly since most lunar endogenic activity ended more than 3 G.y. ago [with several important exceptions; see Hiesinger et al. (2000)]. Thus, over the last 3 G.y. or so, impacts alone have dominated modifications to the lunar landscape. Moreover, space missions have studied the Moon extensively. Returned samples of lunar landing sites give us a unique opportunity to ascribe an age to craters and regions where accumulated impact craters have been meticulously counted (see Stöffler and Ryder, 2001; Neukum et al., 2001). Hence, on the Moon we can estimate the cratering rate to be the number of craters of a given diameter accumulated at a given surface during a given time interval.

We start by assuming an ideal case (i.e., where a planetary surface erased by some process begins to accumulate craters). Before the crater degradation/obliteration processes change the population of these craters, the crater SFD reflects the production SFD or "the standard distribution" of the projectiles. To understand this production function, many authors have tabulated and generalized a huge amount of data on lunar crater counts. In this chapter, we will concentrate on the lunar "production" crater SFD proposed by W. Hartmann and G. Neukum (see Neukum et al., 2001).

\subsection{Hartmann Production Function (HPF)}

To represent the crater SFD found on the terrestrial planets, Hartmann uses a log-incremental SFD representation with a standard diameter bin size. We call his result the "Hartmann production function," or HPF. The number of craters per kilometers squared is calculated for craters in the diameter bin $D_{L}<D<D_{R}$, where $D_{L}$ and $D_{R}$ are the left and right bin boundary and the standard bin width is $\mathrm{D}_{\mathrm{R}} / \mathrm{D}_{\mathrm{L}}=2^{1 / 2}$.

The tabulated HPF is an assemblage of data selected by Hartmann to present the production function for one specific moment of time: the average time of lunar mare sur-
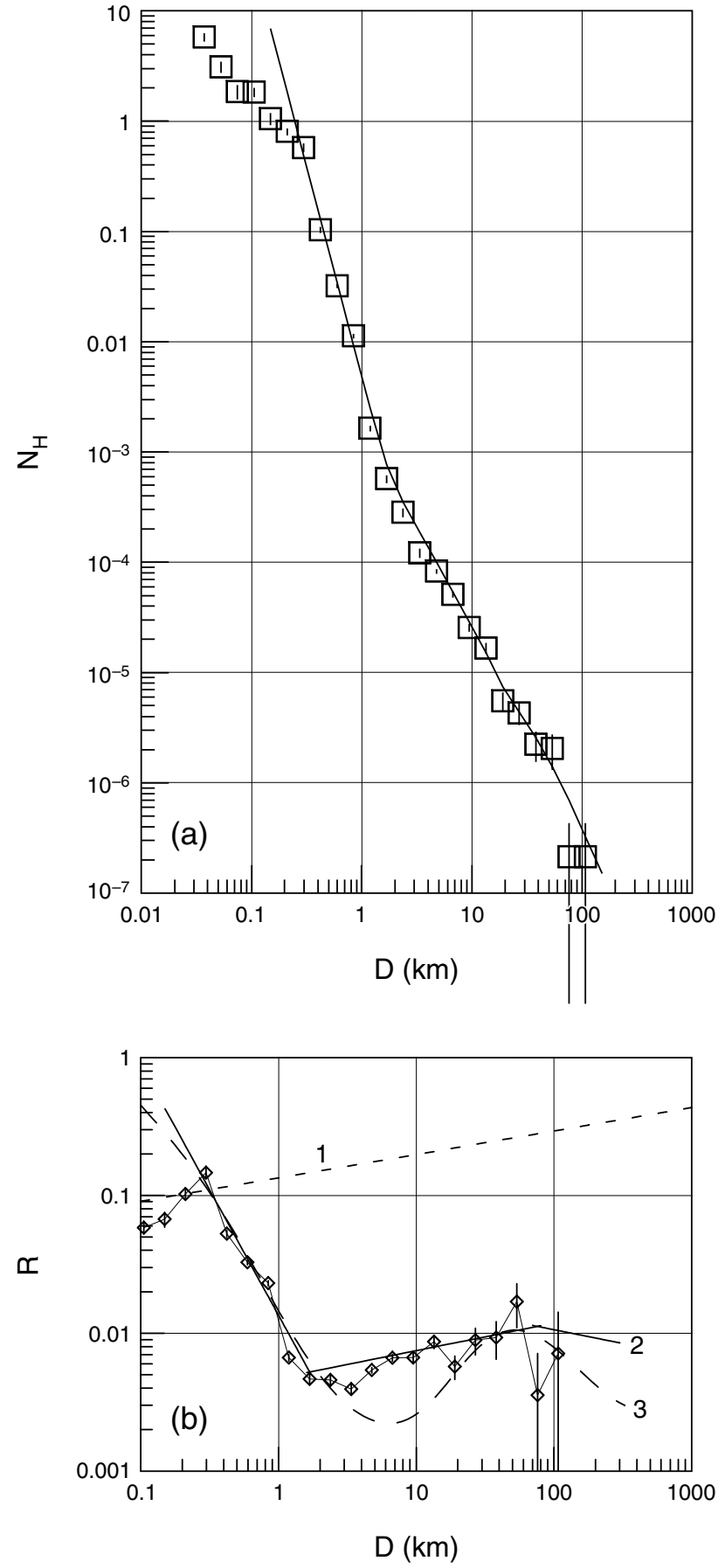

Fig. 1. (a) The incremental representation of the Hartmann production function (HPF). The HPF, in a direct sense, is the set of points shown in the plot. Straight lines represent the piece-wise power law fitting to the data (equation (1)). (b) Comparison of production functions derived by Hartmann (HPF) and Neukum (NPF) in the $\mathrm{R}$ plot representation. The maximum discrepancy between HPF (2) and NPF (3) (roughly a factor of 3) is observed in the diameter bins around $\mathrm{D} \sim 6 \mathrm{~km}$. Below $\mathrm{D} \sim 1 \mathrm{~km}$ and in the diameter range of $30-100 \mathrm{~km}$, the HPF and NPF give the same or similar results. Fitting the HPF to equation (3), we obtain a model age of 3.4 G.y. The NPF, which is fit to the wide range count of impact craters in the Orientale Basin, yields a model age of $~ 3.7$ G.y. The dashed line 1 represents the approximate saturation level estimated by Hartmann (1995). 
TABLE 1. Coefficients in equation (2).

\begin{tabular}{lcccc}
\hline & "Old" N(D) & $\begin{array}{c}\text { "New" N(D) } \\
\text { (Neukum, 1983) }\end{array}$ & $\begin{array}{c}\text { "New" N(D) } \\
\mathrm{a}_{\mathrm{i}}\end{array}$ & $\begin{array}{c}\mathrm{R}(\mathrm{D}) \text { for Projectiles } \\
\text { (Ivanov et al., 2001) }\end{array}$ \\
\hline $\mathrm{a}_{0}$ & -3.0768 & -3.0876 & & - \\
$\mathrm{a}_{1}$ & -3.6269 & -3.557528 & $\pm 3.8 \%$ & +1.375 \\
$\mathrm{a}_{2}$ & +0.4366 & +0.781027 & $\pm 3.9 \%$ & +0.1272 \\
$\mathrm{a}_{3}$ & +0.7935 & +1.021521 & $\pm 2.5 \%$ & -1.2821 \\
$\mathrm{a}_{4}$ & +0.0865 & -0.156012 & $\pm 1.6 \%$ & -0.3075 \\
$\mathrm{a}_{5}$ & -0.2649 & -0.444058 & $\pm 0.88 \%$ & +0.4149 \\
$\mathrm{a}_{6}$ & -0.0664 & +0.019977 & $\pm 1.3 \%$ & +0.1911 \\
$\mathrm{a}_{7}$ & +0.0379 & +0.086850 & $\pm 0.78 \%$ & -0.04261 \\
$\mathrm{a}_{8}$ & +0.0106 & -0.005874 & $\pm 1.8 \%$ & -0.03976 \\
$\mathrm{a}_{9}$ & -0.0022 & -0.006809 & $\pm 1.8 \%$ & $-3.1802 \times 10^{-3}$ \\
$\mathrm{a}_{10}$ & $-5.18 \times 10^{-4}$ & $+8.25 \times 10^{-4}$ & $\pm 5.6 \%$ & $+2.799 \times 10^{-3}$ \\
$\mathrm{a}_{11}$ & $+3.97 \times 10^{-5}$ & $+5.54 \times 10^{-5}$ & $\pm 24.1 \%$ & $+6.892 \times 10^{-4}$ \\
$\mathrm{a}_{12}$ & - & - & - & $+2.614 \times 10^{-6}$ \\
$\mathrm{a}_{13}$ & - & - & - & $-1.416 \times 10^{-5}$ \\
$\mathrm{a}_{14}$ & - & - & - & $-1.191 \times 10^{-6}$ \\
\hline
\end{tabular}

* "Sensibility" is the coefficient variation that changes the N(D) value a factor of 2 up and down.

face formation. Here the condition to have a fresh surface is satisfied by the fact that most lunar mare basalt samples have a narrow range of ages [e.g., 3.2-3.5 Ga (Stöffler and Ryder, 2001); note that some lava flows may be younger (see Hiesinger et al., 2000)]. Hence, the age variation is represented by a factor of 1.1 .

The tabulated HPF has been constructed by combining and averaging crater counts in different areas of the Moon. For this reason, it should be treated as a relatively reliable model to deduce the projectile production function. The HPF, in incremental form, takes the form of a piece-wise three-segment power law (Hartmann, 1995; see also Ivanov, 2001)

$$
\begin{gathered}
\log \mathrm{N}_{2^{1 / 2}}=-2.616-3.82 \log \mathrm{D}_{\mathrm{L}} \\
\mathrm{D}<1.41 \mathrm{~km} \\
\log \mathrm{N}_{2^{1 / 2}}=-2.920-1.80 \log \mathrm{D}_{\mathrm{L}} \\
1.41 \mathrm{~km}<\mathrm{D}<64 \mathrm{~km} \\
\log \mathrm{N}_{2^{1 / 2}}=\begin{array}{l}
-2.198-2.20 \log \mathrm{D}_{\mathrm{L}} \\
\mathrm{D}>64 \mathrm{~km}
\end{array}
\end{gathered}
$$

This function is shown in Fig. 1. Hartmann's choice of power-law segments was made in the 1960s when this work was begun. Note that some selections were for historical reasons; at that time, only the shallow branch with $1.41 \mathrm{~km}<$ $\mathrm{D}<64 \mathrm{~km}$ was well established, with the preexisting literature suggesting various laws for asteroids and meteorites that Hartmann was attempting to relate to lunar data.

\subsection{Neukum Production Function (NPF)}

In a series of publications, Neukum [for summaries, see Neukum (1983) and Neukum and Ivanov (1994)] proposed an analytical function to describe the cumulative SFD of lunar impact craters. He showed that the production function had been more or less stable from Nectarian to Copernican epochs (i.e., from practically more than 4 G.y. ago until now). By this time the full size spectrum of craters was known, and in contrast to the piece-wise exponential equations used for the HPF, Neukum computed a polynomial fit to the cumulative number of craters, N, per kilometers squared with diameters larger than a given value $\mathrm{D}$. For the time period of 1 G.y., N(D) may be expressed (Neukum, 1983) as

$$
\log _{10}(N)=a_{0}+\sum_{n=1}^{11} a_{n}\left[\log _{10}(D)\right]^{n}
$$

where $\mathrm{D}$ is in $\mathrm{km}, \mathrm{N}$ is the number of craters with diameters $>$ D per $\mathrm{km}^{2}$ per G.y., and the coefficients $\mathrm{a}_{\mathrm{n}}$ are given in Table 1. Equation (2) is valid for D from $0.01 \mathrm{~km}$ to $300 \mathrm{~km}$.

Recently, the NPF was slightly reworked in the largestcrater part by careful remeasuring in the size range (Ivanov et al., 1999, 2001; Neukum et al., 2001). The time dependence of the $\mathrm{a}_{0}$ coefficient is discussed in the following section 2.3.

A similar equation is used here to present the projectile SFD derived below. Coefficients for this projectile SFD are also listed in Table 1. In the projectile SFD column, the first coefficient $\mathrm{a}_{0}$ has been set to zero for simplicity. This coefficient determines the absolute number of projectiles. The absolute value of $\mathrm{a}_{0}$ for projectiles may be found by fitting to observational data (see Fig. 5 below).

\subsection{Toward a Unified Production Function}

In Fig. 1b, the NPF and HPF are shown in an R plot together with selected data for crater counts on the lunar 
maria and Orientale Basin. The NPF was fit to the crater counts using an assumed age. We find that both the HPF and NPF are a good match to the observational data below $\mathrm{D} \sim 1 \mathrm{~km}$. However, for D > $1 \mathrm{~km}$, the HPF goes well above the NPF, meeting again the NPF at crater diameters D $40 \mathrm{~km}$. A maximum discrepancy of a factor of 3 between HPF and NPF is observed in the diameter bins around D $6 \mathrm{~km}$. Below D $1 \mathrm{~km}$ and in the $30<\mathrm{D}<100 \mathrm{~km}$ range, the HPF and NPF give the same or similar results.

Although Fig. 1b shows that the HPF and NPF share some similarities, the discrepancy of a factor of 3 for $2<$ $\mathrm{D}<20 \mathrm{~km}$ craters requires further investigation. In general, one should be cautious in interpreting data in this range, particularly since different datasets (including the University of Arizona Arthur Crater Catalog from the 1960s) show somewhat different SFD curvatures. We believe that additional studies of lunar mare data will be needed to further refine the accuracy of the main production function curve.

To use a production function, one should first select a portion of the lunar surface where all the accumulated craters since the last resurfacing event can be counted. Examples of such "time slices" are (1) the Orientale Basin, which erased a large area near the base of the Imbrian stratigraphic horizon; (2) the emplacement of mare basalts (Hartmann, 1970; Hartmann et al., 1981); (3) Eratosthenian-aged craters, which mostly have good stratigraphic dates (Wilhelms et al., 1987); and (4) crater rays, which in some cases have a limited lifetime and thus mark an approximate time horizon.

Using the NPF, we show crater counts for lunar areas that differ by a factor of 100 in crater area density (Fig. 2). An examination of these "time slices" suggests that we cannot rule out the simple hypothesis that the lunar production function had a constant shape from $\sim 4 \mathrm{Ga}$ (lunar

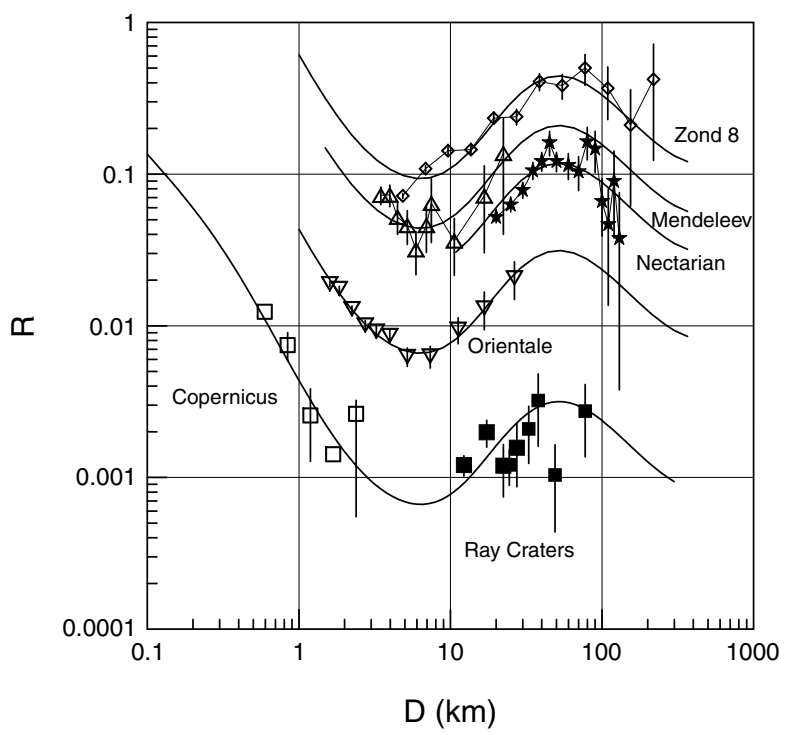

Fig. 2. R plot for several "time slices" of the lunar impact chronology fitted with the NPF curve (equation (2)). See data description in Neukum et al. (2001). highland formation) to $\sim 1 \mathrm{Ga}$ (ray craters). Thus, within the limits of data accuracy, we can assume that the projectile SFD was stable over this interval. To check this hypothesis, we will compare lunar data to those cratering records found on other planets and asteroids.

Although the HPF and NPF have some differences, both assume that the general shape of the SFD striking the Moon over the last 4 G.y. was the same. A different point of view is given by R. Strom (Strom and Neukum, 1988; Strom et al., 1992), who claims that the "modern" (postmare) production function is quite different from that produced during the epoch of the late heavy bombardment. A more extensive treatment of this subject can be found in Strom and Neukum (1988).

\section{CRATERS ON TERRESTRIAL PLANETS}

Taking the "theoretical" lunar SFD described in the previous section, we find it useful to scale it to the other terrestrial planets and compare our results with previously published measurements for selected areas on Mercury, Venus, Earth, and Mars. As discussed in more detail by Hartmann (1977), this procedure requires that we derive the average impact velocity for each planetary body and then determine the average projectile size needed to make a given size crater. The issue of converting between projectiles and craters via a crater-scaling law is discussed elsewhere (e.g., Ivanov, 2001).

\subsection{Average Impact Velocity}

To compute the average impact velocity of PCAs striking various terrestrial planets, we first need an estimate of the orbital distribution of the PCA population. Assuming that the PCA population is well represented by (1) observed asteroids taken from the "astorb.dat" catalog provided by E. Bowell (http://naic.edu/ nolan/astorb.html) and (2) in the case of Earth, by the debiased model population of Earthcrossing objects estimated by Rabinowitz (1993), we can compute impact velocities for various planets using an extended Öpik method (Wetherill, 1967). We recognize that the use of either dataset has some limitations. For (1), we assumed that the observed population of large PCAs did a reasonable job of sampling the debiased population. For (2), we assumed that the sparseness of data points in Rabinowitz's model would not affect our results.

Overall, we found that the average impact velocities of asteroids striking Earth from (1) and (2) were similar, although somewhat higher velocities were found for the debiased population of Earth-crossers. Specifically, the asteroid catalog gives the average impact velocity of $18.6 \mathrm{~km} \mathrm{~s}^{-1}$ (for all asteroids with absolute magnitude $\mathrm{H}<15$ ), while estimates from Rabinowitz's model yielded $20.2 \mathrm{~km} \mathrm{~s}^{-1}$. We believe this difference is a byproduct of peculiar sampling among the $\mathrm{H}<15$ objects; model results suggest that a substantial fraction of undetected Earth-crossing asteroids with $\mathrm{H}<15$ have semimajor axes a $>2$ AU, large eccentricities, and/or high orbital inclinations (Bottke et al., 2000, 2002a; Stuart, 2001; Morbidelli et al., 2002). These values 

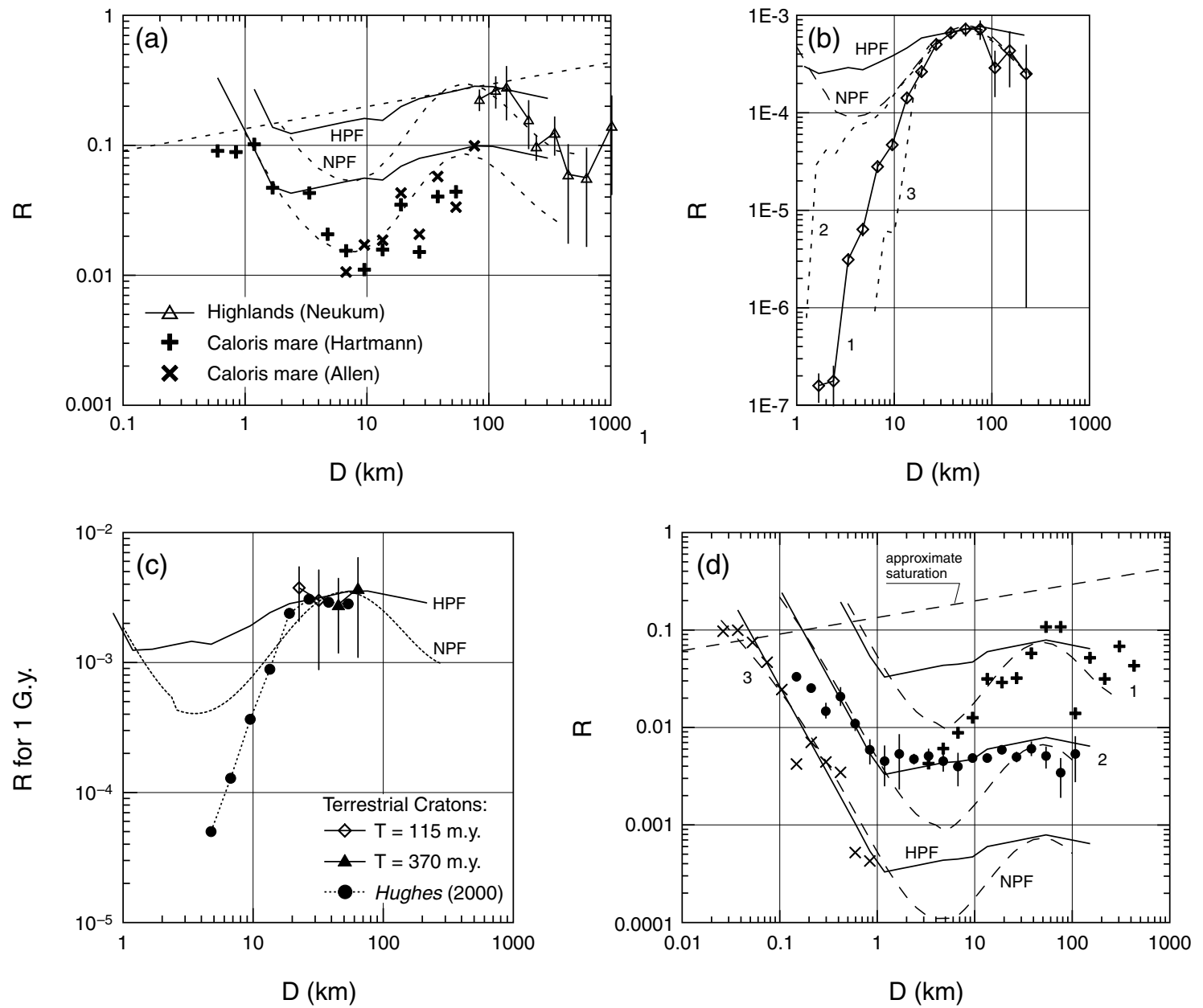

Fig. 3. (a) Crater counts for Mercury's highlands and Caloris mare basin compared with the "lunar analog" curves constructed with HPF and NPF. Dashed lines show an approximate saturation level after Hartmann (1995). Crater counts are digitized from figures in Hartmann et al. (1981). (b) R plot for the size-frequency distribution of venusian craters (1) in comparison with the lunar curve recalculated for venusian conditions with the Schmidt and Housen (1987) scaling law and Croft's (1985) crater collapse model. Dashed curves 2 and 3 represent two models of how projectiles undergo atmospheric disintegration (Ivanov et al., 1997). "Lunar analogs" for an atmosphere-less Venus are shown as HPF and NPF. (c) The R plot for terrestrial craters in comparison with data for cratons (North American + European). To determine the change in the impactor flux over time, the two datasets are divided by factors of 0.115 (115 m.y.) and 0.370 (370 m.y.) in order to put them at the 1-G.y. position. Black dots are crater counts provided by Hughes (2000). (d) R plot for martian craters. The crater SFD for heavily cratered terrain [1, after Hartmann et al. (1981)], "young plains" (2, after Strom et al. (1992)] and a relatively younger volcanic caldera floor (Hartmann et al., 1999a). Dashed lines show an approximate saturation level after Hartmann (1995).

also account for gravitational focusing, which decreases with increasing encounter velocity. Hence, different model assumption about encounter velocities result in variation of impact rate comparisons for the terrestrial planets.

\subsection{Projectile Sizes}

Using scaling laws and estimated impact velocities, one can find the projectile $\mathrm{SFD}, \mathrm{dN} / \mathrm{dD}_{\mathrm{P}}$, for a given impactcrater SFD, dN/dD. To simplify the problem in this chapter, we test the endmember hypothesis of a purely asteroidal projectile flux onto the terrestrial planets. Having such an estimate, we keep open the problem of the cometary impact fraction in the observed crater population. For the same reason, we assume the same projectile density $\left[2.7 \mathrm{~g} \mathrm{~cm}^{-3}\right.$, the density of typical S-type asteroids (Britt et al., 2002)] for all estimates. The procedure we use to transform craters into projectiles (and vice versa) can be found in Ivanov et al. (2001). Briefly, the crater-scaling law derived by Schmidt and Housen (1987) is used to estimate the transient and simple crater diameter for a given projectile. The collapse of complex craters (e.g., Melosh and Ivanov, 1999) is taken into account using the semiempirical model derived by Croft (1985) (see also Chapman and McKinnon, 1986; McKinnon et al., 1991).

The SFD of the projectiles is approximated in the same form as equation (2), with a polynomial of 14th degree valid for projectile diameters between $\sim 0.25 \mathrm{~m}$ and $\sim 27 \mathrm{~km}$. Com- 
puting the largest projectile sizes from the largest craters, however, is more complicated. The SFD for large craters is based on the lunar basins, all of which are very old and do not appear in younger crater populations. Moreover, the basin assignment of a crater diameter $\mathrm{D}$ to a given basin involves interpretation of the origin of multiring structures.

Polynomial coefficients for the R plot are listed in Table 1. The estimated projectile SFD is used below to produce a model ("lunar analog") for Mercury, Venus, Earth, and Mars. This SFD, shown graphically in Fig. 3, is also compared with the recent data on the main-belt SFD (Fig. 4).

\subsection{Cratering Records}

In this section, we apply the lunar-based projectile SFD to each terrestrial planet, accounting for the specific impact velocity and gravity of each planetary body. Our results are summarized in Fig. 3, which shows crater counts from various regions of Mercury, Venus, Earth, and Mars compared to the "lunar analog" SFD curve.

3.3.1. Mercury. The mare surface in the Caloris Basin is one of the few areas suitable for production function measurements of small- to intermediate-sized craters on Mercury. Figure 3a compares direct measurements and calculated SFD (the "lunar analog"). The good coincidence of these data shows a definite similarity of projectile SFDs on the Moon and Mercury in the projectile diameter range from 1 to $\sim 100 \mathrm{~km}$, with a steep part for smaller craters and the " $\mathrm{R}$ minimum" for $\sim 8-\mathrm{km}$ craters. However, the age of Caloris Basin is comparable to the age of Orientale Basin.

3.3.2. Venus. Magellan data allow us to compare the lunar data averaged over the last 3 G.y. with a planetary surface with an age of $\sim 0.5 \mathrm{Ga}$. The presence of the atmosphere may be taken into account using a model of projectile atmospheric passage [i.e., model results for both Venus and Titan can be found in Ivanov et al. (1997)]. The resulting comparison (Fig. 3b) suggests that venusian craters were formed by a projectile population with a similar SFD for $\mathrm{D}>10 \mathrm{~km}$ (projectile diameters $\mathrm{D}_{\mathrm{P}}>2 \mathrm{~km}$ ). The $\mathrm{R}$ maximum at D 50-70 km exists both on the Moon (3-4 G.y.) and on Venus ( $\sim .5$ G.y.). Based on these results, we conclude that the corresponding $\mathrm{R}$ maximum in the projectile distribution in the range of $\mathrm{D}_{\mathrm{P}} \sim 5 \mathrm{~km}$ is stable in time.

We point out that our model of venusian atmospheric entry and projectile destruction is based on the work described in Ivanov et al. (1997). McKinnon et al. (1997), on the other hand, present a different model of atmospheric shielding that also reproduces venusian crater counts, even though they use a simple power-law SFD for projectiles. The disagreement between the two models needs to be studied more thoroughly. Here we only note that Ivanov et al. (1997) derive their results from numerical simulations of stony bodies striking Venus' atmosphere, while McKinnon et al. (1997) use analytical estimates.

3.3.3. Earth. Hartmann $(1965,1966)$ pointed out that large terrestrial craters reflect an older population, while smaller craters are continually removed by erosion, produc- ing an observed SFD that differs from the production function. The inspection of data from the North American and European cratons (Grieve and Shoemaker, 1994) suggests that it is possible to distinguish two populations of craters: (1) eight craters with diameters from 24 to $39 \mathrm{~km}$, the oldest being 115 m.y. old; and (2) eight craters with diameters from 55 to $100 \mathrm{~km}$, with the oldest being $370 \mathrm{~m}$.y. old. The oldest age in each set gives an estimate of the accumulation time. For a proper balance between crater diameter bin width and the number of craters per bin, only two bins for each age subpopulation are used to represent the crater production rate.

We assume that craters smaller than $\sim 20 \mathrm{~km}$ in the younger set and smaller than $\sim 45 \mathrm{~km}$ in the older set are depleted by erosion. Figure $3 \mathrm{c}$ shows the R plot of terrestrial data recalculated to the reference age of $1 \mathrm{Ga}$ assuming a constant crater production rate $(\mathrm{R}$ values are divided by 0.115 and 0.370 respectively). The assumption of a constant crater production rate does not appear to contradict the lunar chronology when it is scaled to Earth. We find that the recent terrestrial production rate estimated by Hughes (2000) yields similar results to our simplified analysis (dark circles in Fig. 3c). The poor statistics for terrestrial craters cannot help us resolve the production function's shape. However, the terrestrial craters do help us constrain variations in the impact rate.

3.3.4. Mars. Several groups have investigated the martian crater record. A good summary of our knowledge from the Viking program can be found in Strom et al. (1992) for craters with D $>8 \mathrm{~km}$, while more recent crater data obtained by Mars Global Surveyor is discussed by Hartmann et al. (1999a,b). A recent review of both datasets can be found in Hartmann and Neukum (2001) for craters down to $\mathrm{D}=11 \mathrm{~m}$. Here we present limited examples of the martian cratering record; in general, they show the same trends as those seen on the other terrestrial planets (Fig. 3d). It is believed that small martian craters have a high obliteration rate, such that any analysis of the data requires one to model how the crater density evolves toward equilibrium. The equilibrium-controlling processes may even vary in different regions or times on Mars. Still, the presence of a $\mathrm{R}$ maximum in the SFD for highlands can be readily observed. The $\mathrm{R}$ minimum in the SFD is less obvious, especially in crater counts published by Strom et al. (1992). We postpone the discussion of this important question for the future.

3.3.5. Craters on asteroids. The Galileo and NEAR Shoemaker spacecraft returned images of four asteroids: Gaspra, Ida, Mathilde, and Eros (Belton et al., 1992, 1994; Chapman et al., 1996a,b; Veverka et al., 1997, 2000). All four bodies are covered by impact craters. Assuming that main-belt asteroids strike one another at average velocities of $5.5 \mathrm{~km} / \mathrm{s}$, it is possible to estimate the small-projectile asteroid SFD from the crater SFD (Ivanov et al., 2001).

We assume that on Gaspra, the impact crater SFD represents the production function. Ida's smaller craters, on the other hand, are believed to be close to saturation (equilibrium); only the largest craters are believed to be below the 
saturation limit (Chapman et al., 1996a). Large craters on Ida may be formed in the gravity regime (Asphaug et al., 1996).

For Mathilde and Eros, we use the published impact crater SFD (Veverka et al., 1997, 2000). The scaling of craters on Mathilde is not well defined due to the unusually low density (high porosity) of the target. As a first-order approximation, we use scaling parameters presented by Schmidt and Housen (1987) for the loosest soil.

Using the assumptions discussed above, it is possible to construct a model projectile distribution for all imaged asteroids (Fig. 4). To simplify things as much as possible, Fig. 4a
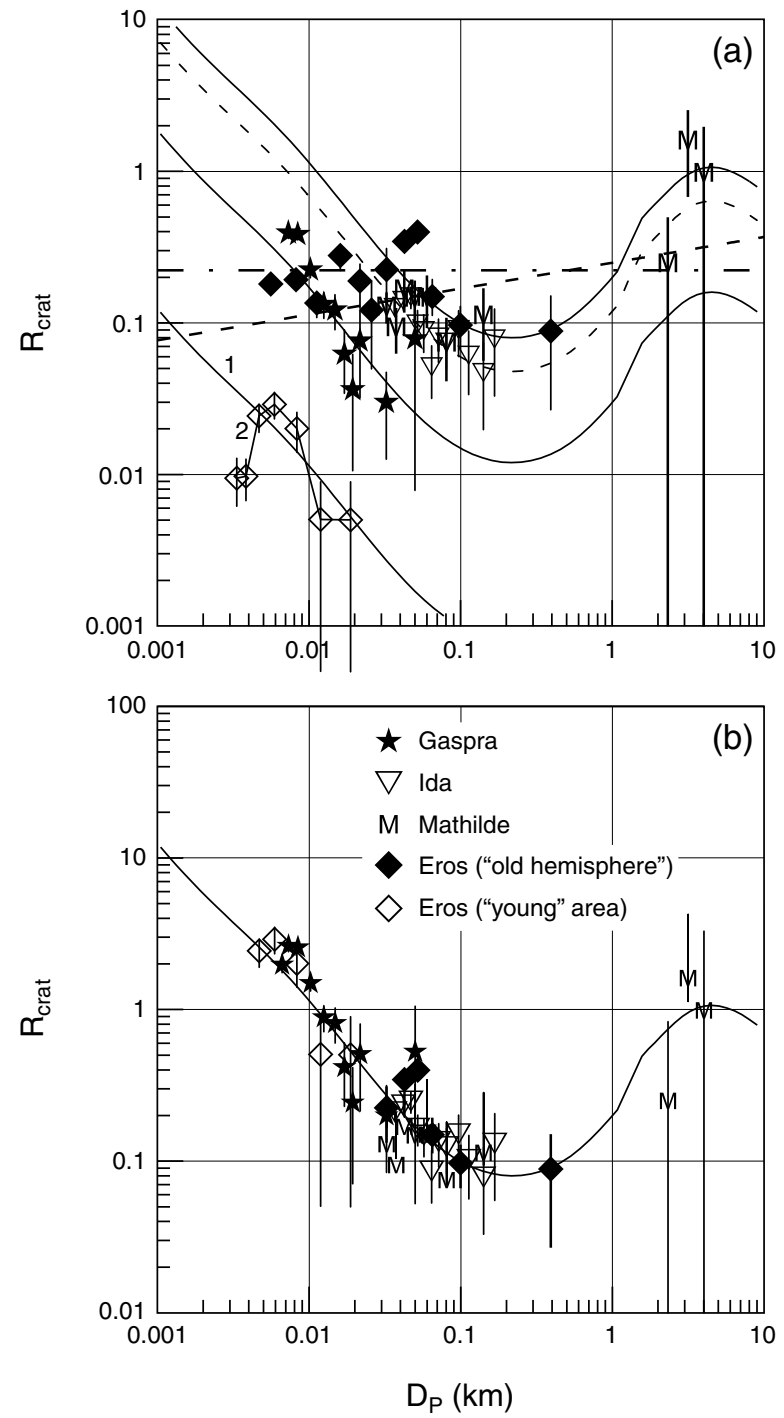

Fig. 4. The R plot for the projectile population derived from craters found on Gaspra, Ida, Mathilde, and Eros. The solid lines represent the projectile SFD derived from lunar cratering records. (a) $\mathrm{R}$ values calculated for craters plotted against their estimated projectile diameter: 1 - geometrical saturation limit; $2-\mathrm{em}$ pirical saturation limit according to Hartmann (1995). (b) Selected data points from (a) below a saturation crater density have been shifted vertically to fit onto a single curve. shows $\mathrm{R}$ values for craters vs. projectile diameter, while Fig. 4b shows all asteroid cratering data (below a saturation limit) fit to a single curve. Using Eros data as a reference level, we conclude that its younger surfaces have a crater density that is a factor of 0.01 less than that of its oldest surfaces (Veverka et al., 2000; Chapman, 2002). On Gaspra, the same ratio is $\sim 0.15$, while it is $\sim 0.6$ for Ida and Mathilde (within the accuracy limits of available scaling laws).

For comparison, the lunar-derived projectile SFD is also shown. The model results for asteroid craters demonstrate the presence of the $\mathrm{R}$ minimum of the projectile SFD curve. We find this minimum is in approximately the same range of projectile diameters as that seen for the near-Earth asteroids (NEAs).

When comparing the cratering records found on the terrestrial planets and asteroids, we found that we could not reject the idea that a single projectile population formed most observed craters. Thus, it seems reasonable to assume that asteroids dominate the population of crater-forming bodies in the inner solar system. The search for deviations from this simple assumption will be left for future work. The interplanetary comparison is consistent with conclusions made by Hartmann (1995), namely that (1) the lunar crater record is consistent with a relatively uniform size distribution of interplanetary impactors stretching back 4 G.y. and (2) this same population has struck all the bodies of the inner solar system.

\section{MAIN-BELT AND NEAR-EARTH ASTEROIDS}

\subsection{Main Belt}

In this section, we compare our projectile SFD with direct astronomical observations of main-belt asteroids. Earthbased astronomical observations and the satellite infrared survey (IRAS) have revealed all main-belt asteroids with diameters larger than about $40 \mathrm{~km}$ (van Houten et al., 1970; Gradie et al., 1989; Cellino et al., 1991). For smaller diameters, one usually assumes a power-law SFD of the form $\mathrm{dN} / \mathrm{dD}_{\mathrm{P}} \sim \mathrm{D}_{\mathrm{P}}^{-\mathrm{k}}$, where the value of $\mathrm{k}$ may vary between 2.95 [the so-called PLS-slope, after the Palomar-Leiden Survey (see van Houten et al., 1970)] up to 3.5 [the so-called Dohnanyi slope (see Dohnanyi, 1969)]. $\mathrm{K}=3.5$ is a typical value for a self-similar cascade of fragments. Davis et al. (1994) used a geometrical average of two possible powerlaw distributions, the PLS distribution and estimations by Cellino et al. (1991), to analyze the IRAS data (Fig. 5).

Deviations from a simple power-law crater SFD, considered above, suggest that the asteroid SFD also deviates from a simple power law at diameters smaller than the completeness limit. For large bodies ( $100 \mathrm{~km}$ in diameter) the non-power-law SFD is thought to be an intrinsic feature of the initial distribution of small bodies before the main belt accumulated (Davis et al., 1985). Hartmann et al. (1999c) pointed out that the Yarkovsky effect may have strong influence in controlling size distribution below $\mathrm{D}_{\mathrm{p}} \sim 10 \mathrm{~m}$, and 

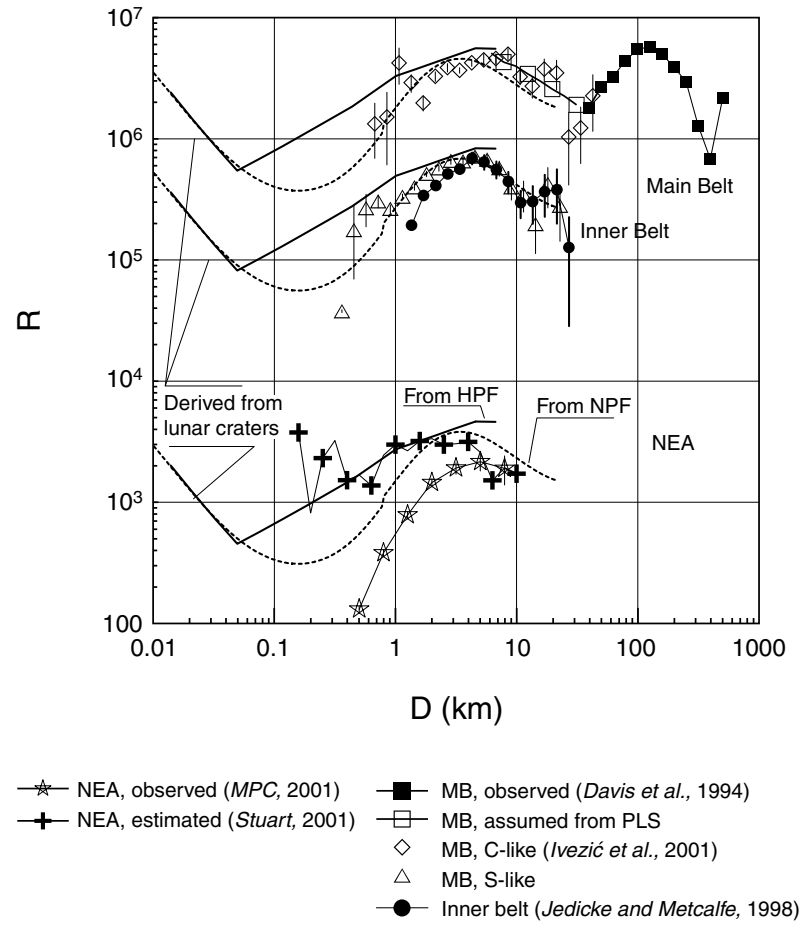

Fig. 5. R plot for main-belt asteroids according to Davis et al. (1994) (filled squares — observed asteroids, open squares - assumed by Palomar-Leiden survey), Spacewatch data by Jedicke and Metcalfe (1998) for all the inner belt (black dots error bars), Sloan digital sky survey data by Ivezić et al. (2001) for C-like (diamonds) and S-like (triangles) asteroids, and the projectile SFDs found using lunar craters according to HPF and NPF. Observed NEAs are shown as stars $(M P C, 2001)$, while crosses are for the LINEAR survey data (Stuart, 2001). The $\mathrm{R}$ value for asteroids is calculated in the same way as that for craters: $R=D_{p}^{3} d N / d_{p}$.

this influence may vary from one asteroid taxonomic type to another (see also Bottke et al., 2002b).

Another possible mechanism for producing such deviations is based on modeling results describing impact evolution in the main belt (i.e., a "wavy" SFD) (Campo Bagatin et al., 1994a,b; Durda et al., 1998; Davis et al., 2002). In a model proposed by Campo Bagatin et al. (1994a,b), "waves" seen in the main-belt SFD are produced when small particles are removed from the collisional cascade. A lack of small fragments result in an increase in the number of larger bodies that would have been destroyed by these projectiles. Durda et al. (1998), using numerical modeling results of catastrophic collisions (e.g., Love and Ahrens, 1996; Melosh and Ryan, 1997; Benz and Asphaug, 1999), found that the transition from strength scaling to gravity scaling was also capable of producing a wave in the main-belt SFD. Occurring for bodies with diameters near a few hundred meters, self-gravity helps prevent catastrophic disruption events by allowing fragments to reaccumulate with the target asteroid. As bodies get stronger via gravity, more projectiles of that size are available to disrupt larger asteroids, ultimately leading to a wave in the SFD.

Jedicke and Metcalfe (1998) published an estimate of the debiased main-belt SFD based on absolute magnitudes measured as part of the Spacewatch survey. The SFD was converted from absolute magnitudes to diameters by Durda et al. (1998) using albedo measurements taken from large asteroids (Gradie et al., 1989). Their SFD estimates are believed to be valid for asteroid diameters large than $2 \mathrm{~km}$. For comparison, the more direct IRAS SFD estimates are considered valid for D $>20 \mathrm{~km}$ (Cellino et al., 1991).

In Fig. 5, data from direct observations (following Davis et al., 1989) and estimations by Jedicke and Metcalfe (1998) are shown. The projectile SFD, determined above using lunar impact craters, can be compared to these data. Our analysis yields the following points: (1) The direct astronomical data show a relative $\mathrm{R}$ minimum at asteroid diameters $\mathrm{D}$ $30-40 \mathrm{~km}$; the depth of this minimum may vary for different semimajor axes a. This minimum corresponds to a lunar impact crater $\sim 300 \mathrm{~km}$ in diameter. The lunar SFD also shows this minimum. In fact, the lunar SFD, which contains basins up to a size of $\sim 1000 \mathrm{~km}$, shows the same rising $\mathrm{R}$ characteristics as the main-belt SFD as it approaches its $\mathrm{R}$ maximum near $\sim 100 \mathrm{~km}$. (2) Asteroid diameters have a second $\mathrm{R}$ maximum at $\mathrm{D} \sim 4-5 \mathrm{~km}$. This maximum is visible in the inner and central main belt $(2.0<\mathrm{a}<2.6 \mathrm{AU})$. It may also exist in the outer main belt, although the needed data is currently beyond our detection limit. The asteroidal $\mathrm{R}$ maximum at $\mathrm{D} \sim 4-5 \mathrm{~km}$ corresponds to a lunar crater diameter of $\sim 50 \mathrm{~km}$. The lunar SFD does show this maximum. (3) For asteroid diameters between 2 and $20 \mathrm{~km}$, the general shape of the SFD of the inner asteroid belt and the SFD derived from lunar impact craters is identical within the error limits of available data.

Based on this evidence, we can make some predictions. Figure 5 shows that the projectile SFD derived from lunar craters has an inflection point near $50 \mathrm{~m}$. We suggest that the SFD of main-belt asteroids - the main source of projectiles for cratering on terrestrial planets - may have a similar inflection point. To test this idea, we will need better and more numerous main-belt observations than are currently available.

\subsection{Near-Earth Asteroids}

A similar SFD is found for NEAs. Figure 6 gives a summary of several recent SFDs estimated from astronomical observations and debiased modeling work of the NEA population. These results are compared to the projectile SFD derived from lunar cratering records with HPF and NPF. The similarity between (1) crater-forming projectiles derived from 1- to 4-G.y.-old surfaces on the Moon, (2) the observed main-belt asteroid population (Fig. 5), and (3) NEAs (Fig. 6) suggests a common connection, namely that the main belt is the predominant source of both the current NEA population and those projectiles that have struck the Moon over the last several billion years. The wavelike shape of 


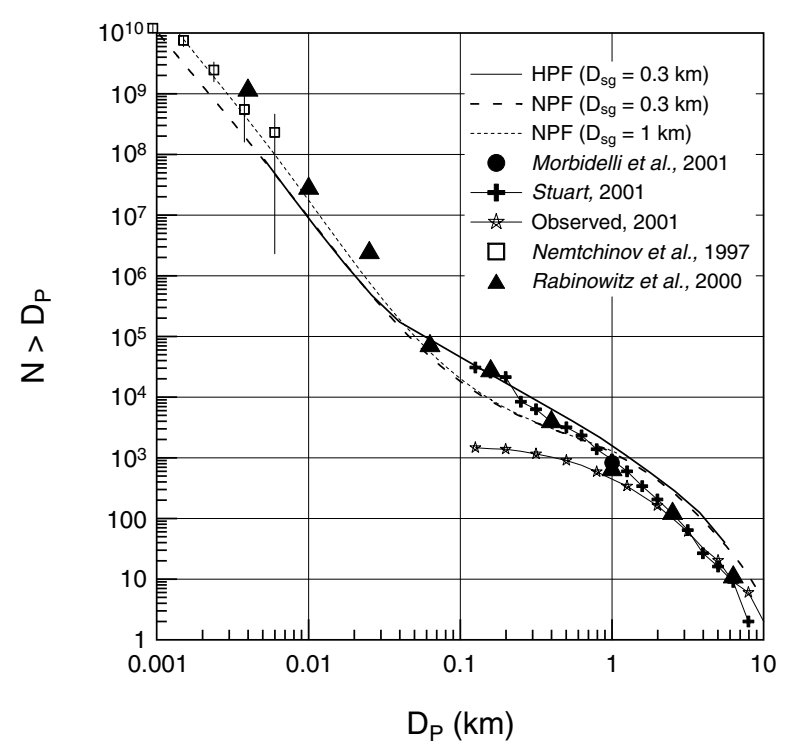

Fig. 6. Estimates of a cumulative, $N>D_{P}$, size-frequency distribution for NEAs. The solid, dashed, and dotted lines are model distributions derived from the HPF and NPF for various assumed strength-to-gravity transition diameters for lunar craters. The absolute position of these curves corresponds to the lunar chronology (equation (3)) combined with estimated average impact probability for Earth-crossing asteroids (ECAs). The number of NEAs (defined as bodies with $\mathrm{q}<1.3 \mathrm{AU}$ ) is larger. For observed bodies with $\mathrm{H}<15$, the ECA to NEA ratio is $\sim 0.57$. Recent astronomical estimates by Rabinowitz et al. (2000), Morbidelli et al. (2001), and Stuart (2001) are generally consistent with these estimates. Satellite observations of bolides entering Earth's atmosphere (Nemtchinov et al., 1997) are consistent with our results for small $\left(\mathrm{D}_{\mathrm{P}}<\right.$ $10 \mathrm{~m}$ ) bodies, though we caution that it is problematic to convert light flashes detected in the atmosphere into projectile sizes. The average probability of ECA impacts is used to estimate the total number of projectiles and the impact (or atmospheric entrance) rate.

the main-belt SFD appears to be a byproduct of collisional processes (Davis et al., 2002). The shape of the NEA population is probably an artifact of both the shape of the mainbelt SFD and the subtle dynamical mechanisms that allow some main-belt asteroids to escape over long timescales [i.e., collisions coupled with the Yarkovsky effect and chaotic resonances (Bottke et al., 2002b; Morbidelli et al., 2002)].

\section{PLANETARY CRATERING RATES}

The balance between the PCA supply and depletion rates controls the abundance of PCAs and, accordingly, the terrestrial planet cratering rate (Morbidelli et al., 2002). Using a conservative approach, we assume that the currently observed population of PCAs has been in steady state over the last 3 G.y. For more ancient periods (i.e., the end of the heavy bombardment period), we assume that the relative cratering rates on the terrestrial planets with respect to the Moon was the same as relative cratering rates for the cur- rently observed PCAs. Each of these assumptions should be used with caution.

Most investigators believe that the cratering projectile flux over the last 3 G.y. was relatively constant (with possible variations within a factor of 2). Prior to 3 G.y., the PCA flux was much larger, though it was certainly decaying with time. This ancient period is commonly named "the Late Heavy Bombardment," or LHB. The LHB decay rate is still considered controversial (Hartmann et al., 2000). In this section, we adopt the values favored by Neukum (1983). Neukum (1983) used lunar rock dating to calibrate the lunar crater count and thus reveal the general character of the bombardment flux decay. His work can be expressed analytically in the form

$$
\begin{gathered}
\mathrm{N}(1)=5.44 \times 10^{-14}(\exp (6.93 \mathrm{~T})-1)+ \\
8.38 \times 10^{-4} \mathrm{~T}
\end{gathered}
$$

with $\mathrm{N}(1)$ the number of craters larger that $1 \mathrm{~km}$ in diameter per $\mathrm{km}^{2}$ and $\mathrm{T}$ being the crater accumulation time (crater retention age) in G.y. Assuming the shape of the SFD is constant in time, equation (3) should be valid for any crater diameter with the proper numerical coefficients.

\subsection{Cratering Rate Comparison}

The estimated lunar cratering rate has been calibrated using returned samples that have been dated. For other planets, we can rely only upon measured impact crater densities. To translate the lunar crater chronology to other planets, one needs to take into account the differences in planetary gravity, number of planet-crossing bodies, and orbital parameters of the potential projectiles, which in turn control their collision probabilities and impact velocities. As an important example, we compare here the crater rate on Mars to that of the Moon. We discuss first the impact rate, defined as the number of impacts of bodies of the same size per unit surface area per unit of time. The Mars/Moon impact rate ratio is named " $\mathrm{R}$ bolide" $\left(\mathrm{R}_{\mathrm{b}}\right)$ following Hartmann (1977). This factor is the basis of the system to date the martian surface - a system highly consistent with martian meteorite age data (Hartmann, 1999).

Gravity and target materials are vary from planet to planet. Hence the "R bolide" ratio does not correspond directly to the cratering rate ratio, $\mathrm{R}_{\mathrm{c}}$. Moreover, for a non-power-law projectile SFD, the cratering rate ratio depends intimately on crater diameter; note that different-sized projectiles can form craters with the same diameter on different planets, such that the projectiles may lie on completely different parts of the production SFD.

Estimates of $R_{b}$ and $R_{c}$ can be found in various ways. Ivanov (2001) followed the technique proposed for comets by Shoemaker and Wolfe (1982): (1) The lunar crater SFDs were used to derive the projectile SFDs; (2) the orbits and sizes of observed asteroids were used to both calibrate the projectile SFDs and compute their associated impact velocities; and (3) these values were inserted into crater-scaling 
laws, which were then used to determine crater-production rates on Mars and the Moon and the values $R_{b}$ and $R_{c}$. The limitation of this "bottom-up" approach is that, so far, observed (and potentially biased) asteroids have been used to determine several important parameters.

The alternative "top-down" approach relies on the recent work by Bottke et al. (2002a) (see also Morbidelli et al., 2002), who constructed a model of the debiased orbital and size distribution of the NEA and Mars-crossing asteroid populations.

\section{2. $\quad \mathbf{R}_{\mathrm{b}}$ from Observed Asteroids}

A quantitative description of the PCA flux requires a good understanding of the physical and dynamical evolution of main-belt asteroids (e.g., Bottke et al., 2002a; Morbidelli et al., 2002) or a method to deduce this information from the observed population. Ivanov (2001) used the latter approach to estimate the PCA flux. His method, originally developed by Shoemaker and Wolfe (1982) to estimate the population of short-period comets, was as follows: Ivanov tabulated the set of observed PCA orbits, sorted them according to perihelion distance, $\mathrm{q}$, and then estimated their average collision probabilities and impact velocities as a function of q. In this way, one uses observed orbits as an assumed representative ensemble of the statistically "averaged" steady-state population of PCAs. In this chapter, we used the February 2002 list of osculating orbits in the smallbody database "astorb.dat" (http://asteroid.lowell.edu).

The observed asteroid population is incomplete. Several techniques to remove observational bias exist (e.g., Rabinowitz, 1993, 1997; Rabinowitz et al., 1994; Jedicke and Metcalfe, 1998; Bottke et al., 2000; Jedicke et al., 2002). For Mercury and Venus, we can make only rough estimates of the cratering rate from the observed asteroid population because many objects with large $(\mathrm{a}, \mathrm{e}, \mathrm{i})$ values have yet to be detected. For Mars, however, the list of observed asteroids allows us to make a simple correction to estimate the Mars/Moon cratering-rate ratio. We use a simple approach to remove the obvious bias in the q-distribution: We sort PCAs by their absolute magnitude and assume that the brightest asteroids represent a nearly-observationally complete set of objects. For now, we assume that the population of Mars-crossers with $\mathrm{H}<15$ bodies is relatively complete [in close agreement with Bottke et al. (2000, 2002a)]. To test the completeness of the $\mathrm{H}<15$ observations, we compared its q distributions for $\mathrm{H}<12$ bodies (Ivanov, 2001). Both N(q) functions appear similar to one another. Note that this procedure cannot be used for NEAs, because the number of $\mathrm{H}<12$ asteroids is less than one. Here we assume that the $\mathrm{q}$ distribution of asteroids with $\mathrm{H}<15$ may be used to estimate $\mathrm{N}(\mathrm{q})$ function for smaller bodies.

For each target (Mars or the Moon) and projectile (PCA), the probability and impact velocity were calculated using an update of Öpik's formulas [refined by Wetherill (1967) for the general case of elliptic orbits both for a target and a projectile]. Öpik's method assumes that the apsides and nodes have random positions. The total collision probability (with a correction factor used to account for observational bias among small Mars-crossers) and the average impact velocity yielded the values needed to compute the cratering rate.

The average impact velocity (over all possible Mars orbit eccentricities) was found to be $\left\langle\mathrm{v}_{\text {Mars }}\right\rangle=9.6 \mathrm{~km} \mathrm{~s}^{-1}$. The Mars/Moon impact rate ratio $\mathrm{R}_{\mathrm{b}}$ averaged in time is $\sim 2$ for asteroids of the same size (Ivanov, 2001).

\section{3. $\quad \mathbf{R}_{\mathrm{b}}$ Estimates from Celestial Mechanics Modeling}

The limitation of the "bottom-up" approach presented in the previous section is that, so far, observed (and potentially biased) asteroids have been used to determine several important parameters. In this section, we check these results using a "top-down" approach. Our computation relies on the recent work by Bottke et al. (2002a) (see also Morbidelli et al., 2002), who constructed a model of the debiased orbital and size distribution of the NEO and intermediate Mars-crossers (IMC) populations. The latter is defined as the population of the asteroids with main-beltlike semimajor axis and inclination, and which intersect the orbit of Mars within a secular cycle of the eccentricity oscillation. Notice that the Bottke et al. model does not incorporate other populations of Mars-crossers, like the Hungarias, Phoceas, and the isotropic comets of Oort Cloud origin, but these are all expected to be secondary sources as far as cratering is concerned.

From this model, we compute the impact rates between our debiased populations and Moon/Mars using the methodology of Bottke et al. (1994). To account for secular variations in Mars/Moon (e, I) values over time, we compute their evolution over the next 10 m.y. using the analytical approximations provided by Laskar (1988). Collision probabilities and mean impact velocities are then computed between these values and a grid of test particles uniformly distributed in (a, e, i) space, with each particle representing a component of the NEA and IMC debiased populations.

Our results indicate the interval between $\mathrm{H}<18$ impacts on the Moon, $\sim 11$ m.y., is over $10 \times$ longer than that for Mars, while the mean impact velocities of material striking the Moon $\left(\sim 19 \mathrm{~km} \mathrm{~s}^{-1}\right)$ is twice that of objects striking Mars at approximately $10 \mathrm{~km} \mathrm{~s}^{-1}$. Thus, using these numbers, we calculate that the ratio of $\mathrm{H}<18$ impactors striking Mars over that of the Moon per $\mathrm{km}^{2}$ per year $\mathrm{R}_{\mathrm{b}}=2.8$.

Computing $\mathrm{R}_{\mathrm{c}}$ requires the conversion of $\mathrm{H}$ into projectile diameter $d$ and crater diameter D. Since the ratio of bright to dark objects in the Mars-crossing and Earth-crossing populations are not well known, and to keep things simple, we will assume that our projectiles have the same intrinsic properties as those described previously in this chapter.

\subsection{Cratering Rate Ratio}

To compute $\mathrm{R}_{\mathrm{b}}$, one needs to take into account the differences between the average impact velocity and surface gravity of Mars and the Moon. Using the modern scaling law for simple craters, we estimate the ratio of crater diam- 
eters produced on Mars and the Moon with an impact of the same projectile to be

$$
\mathrm{D}_{\mathrm{M}} / \mathrm{D}_{\mathrm{m}}=\left(\left\langle\mathrm{v}_{\text {Mars }}\right\rangle /\left\langle\mathrm{v}_{\text {Moon }}\right\rangle\right)^{\beta}\left(\mathrm{g}_{\mathrm{M}} / \mathrm{g}_{\mathrm{m}}\right)^{-\gamma}
$$

where exponents $\beta=0.43$ and $\gamma=0.22$, according to Schmidt and Housen (1987), and subscripts $\mathrm{M}$ and $\mathrm{m}$ are for Mars and the Moon respectively. Ivanov (2001) estimated the $\left\langle v_{\text {Mars }}\right\rangle\left\langle\left\langle v_{\text {Moon }}\right\rangle\right.$ ratio to be $(9.6 / 16.1) \approx 0.6$. The velocity term in equation (4) consequently yields a factor of 0.8 . In Bottke's estimate from section 5.3, the ratio $\left\langle\mathrm{v}_{\text {Mars }}\right\rangle /\left\langle\mathrm{v}_{\text {Moon }}\right\rangle=(10 /$ $19)=0.53$, and the velocity term in equation (4) is 0.76 . The gravity term in equation (4) is the same for both models: $(3.69 / 1.62)^{-0.22} \approx 0.83$. The total difference in $\mathrm{D}_{\mathrm{M}} / \mathrm{D}_{\mathrm{m}}$ ratio in two discussing models is rather small: 0.5 for Ivanov's estimates vs. 0.63 for Bottke's estimate.

If $\mathrm{N}(>\mathrm{D})$ curves on both planetary bodies are compared for the same (steep or shallow) power slope $\left[N(>D) \sim D^{-b}\right]$, the cratering rate ratio is expressed as

$$
\mathrm{R}_{\mathrm{c}}=\left(\mathrm{D}_{\mathrm{M}} / \mathrm{D}_{\mathrm{m}}\right)^{\mathrm{b}} \mathrm{R}_{\mathrm{b}}
$$

For a steep branch of HPF (equation (1a)) b = 3.82 for craters below $\sim 1.4 \mathrm{~km}$ in diameter. The same slope is given by the NPF (see Fig. 1b). For a shallow branch (D > $1.4 \mathrm{~km}$ ) $\mathrm{b}=1.8$ (equation (1b)). For $\mathrm{D}<5 \mathrm{~km}$, one can ignore crater modification issues (see details in Ivanov, 2001). Consequently, the cratering rate ratio is estimated as $R_{c}=0.97$ for $\mathrm{b}=1.8$ and $\mathrm{R}_{\mathrm{c}}=0.43$ for $\mathrm{b}=3.82$ from Ivanov (2001) and $R_{c}=1.22$ for $b=1.8$ and $R_{c}=0.49$ for $b=3.82$ from Bottke's model. Hence the difference in $\mathrm{R}_{\mathrm{b}}$ of $40 \%$ (2.8 vs. 2 ) is reduced to the difference in $R_{c}$ of $27 \%$ for $b=1.8$ and $14 \%$ for $b=3.82$. We conclude that the accuracy of our " $R$ bolide" value is formally improved for our "R crater" value.

The work above suggests that the shape of the projectile SFD striking Mars and the Moon must be understood before the martian and lunar crater rates can be compared to one another. In terms of impact rates, it appears that the proximity of Mars to the asteroid belt (more available projectiles) is partially compensated by its smaller average impact velocity and larger surface gravity. For example, although a given size projectile may strike Mars 2-4x more frequently than the Moon, it generally creates a smaller crater. Consequently, the Mars/Moon cratering rate ratio varies from 0.4 to 1.6 , depending on the steepness of the projectile's SFD (Ivanov, 2001).

\section{CONCLUSIONS}

1. For the last $\sim 4$ G.y., we claim that the shape of production function on the Moon has not changed within the limits of observational accuracy for craters below at least $300 \mathrm{~km}$ in diameter.

2. Application of cratering scaling laws allow us to estimate the size-frequency distribution (SFD) of projectiles from the measured SFD of lunar impact craters. The estimated projectile SFD has a complex form with wavy deviations from a simple power law.
3. The estimated SFD of projectiles allows us to reproduce the impact crater SFD on the terrestrial planets. We conclude that a single projectile population formed the majority of impact craters.

4. The projectile SFD derived from the lunar crater population is similar to the SFD of asteroids in the main asteroid belt (within the limits of accuracy of available data). Within the same limits of accuracy, it appears that the contribution of comets to crater formation in the inner solar system either replicates the wavy SFD seen for asteroids or is relatively insignificant. We believe that main-belt asteroids have provided most of the crater-forming objects striking the terrestrial planets, and that these projectiles belong to a collisionally evolved family of objects.

Acknowledgments. We thank W. McKinnon and R. Strom for helpful reviews. B.I. is supported by the Russian Foundation for Basic Science (RFBR) project \#01-05-64564-a. W.F.B would like to acknowledge support from NASA's Planetary Geology and Geophysics (NAG5-10331), NEO Observations (NAG6-9951), and Mars Data Analysis (NAG5-10603) programs.

\section{REFERENCES}

Asphaug E., Moore J. M., Morrison D., Benz W., Nolan M. C., and Sullivan R. J. (1996) Mechanical and geological effects of impact cratering on Ida. Icarus, 120, 158-184.

Belton M. J. S., Veverka J., Thomas P., Helfenstein P., Simonelli D., Chapman C. R., Davies M. E., Greeley R., Greenberg R., and Head J. W. III (1992) Galileo encounter with 951 Gaspra first pictures of an asteroid. Science, 257, 1647-1652.

Belton M. J. S., Chapman C. R., Veverka J., Klaasen K. P., Harch A., Greeley R., Greenberg R., Head J. W. III, McEwen A., Morrison D., Thomas P. C., Davies M. E., Carr M. H., Neukum G., Fanale F. P., Davis D. R., Anger C., Gierasch P. J., Ingersoll A. P., and Pilcher C. B. (1994) First images of asteroid 243 Ida. Science, 265, 1543.

Benz W. and Asphaug E. (1999) Catastrophic disruptions revisited. Icarus, 142, 5-20.

Bottke W. F., Nolan M. C., Greenberg R., and Kolvoord R. A. (1994) Collisional lifetimes and impact statistics of near-Earth asteroids. In Hazards Due to Comets and Asteroids (T. Gehrels and M. S. Matthews, eds.), pp. 337-357. Univ. of Arizona, Tucson.

Bottke W. F., Jedicke R., Morbidelli A., Petit J. M., and Gladman B. (2000) Understanding the distribution of near-Earth asteroids. Science, 288, 2190-2194.

Bottke W. F., Morbidelli A., Jedicke R., Petit J. M., Levison H. F., Michel P., and Metcalfe T. S. (2002a) Debiased orbital and absolute magnitude distribution of the near Earth objects. Icarus, 156, 399-433.

Bottke W. F. Jr., Vokrouhlický D., Rubincam D. P., and Brož M. (2002b) The effect of Yarkovsky thermal forces on the dynamical evolution of asteroids and meteoroids. In Asteroids III (W. F. Bottke Jr. et al., eds.), this volume. Univ. of Arizona, Tucson.

Britt D. T., Yeomans D., Housen K., and Consolmagno G. (2002) Asteroid density, porosity, and structure. In Asteroids III (W. F. Bottke Jr. et al., eds.), this volume. Univ. of Arizona, Tucson.

Campo Bagatin A., Cellino A., Davis D. R., Farinella P., and Paolicchi P. (1994a) Wavy size distribution for collisional sys- 
tems with a small-size cutoff. Planet. Space Sci., 42, 10491092.

Campo Bagatin A., Farinella P., and Petit J.-M. (1994b) Fragment ejection velocities and the collisional evolution of asteroids. Planet. Space Sci., 42, 1099-1107.

Cellino A., Zappalà V., and Farinella P. (1991) The asteroid size distribution from IRAS data. Mon. Not. R. Astron. Soc., 253, 561-574.

Chapman C. R. (2002) Cratering on asteroids from Galileo and NEAR Shoemaker. In Asteroids III (W. F. Bottke Jr. et al., eds.), this volume. Univ. of Arizona, Tucson.

Chapman C. R. and McKinnon W. B. (1986) Cratering of planetary satellites. In Satellites (J. A. Burns and M. S. Matthews, eds.), pp. 492-580. Univ. of Arizona, Tucson.

Chapman C. R., Ryan E. V., Merline, W. J., Neukum G., Wagner R., Thomas P. C., Veverka J., and Sullivan R. (1996a) Cratering on Ida. Icarus, 120, 77-86.

Chapman C. R., Veverka J., Belton M., Neukum G., and Morrison D. (1996b) Cratering on Gaspra. Icarus, 120, 231-245.

Croft S. K. (1985) The scaling of complex craters. J. Geophys. Res., 90, 828-842.

Davis D. R., Chapman C. R., Weidenschilling S. J., and Greenberg R. (1985) Collisional history of asteroids: Evidence from Vesta and the Hirayama families. Icarus, 62, 30-35.

Davis D., Weidenshilling S. J., Farinella P., Paolicchi P. and Binzel R. P. (1989) Asteroid collisional history: Effects on sizes and spins. In Asteroids II (R. P. Binzel et al., eds.), pp. 805-826. Univ. of Arizona, Tucson.

Davis D. R., Ryan E. V., and Farinella P. (1994) Asteroid collisional evolution: Results from current scaling algorithm. Planet. Space. Sci., 43, 599-610.

Davis D. R., Durda D. D., Marzari F., Campo Bagatin A., and Gil-Hutton R. (2002) Collisional evolution of small-body populations. In Asteroids III (W. F. Bottke Jr. et al., eds.), this volume. Univ. of Arizona, Tucson.

Dohnanyi J. W. (1969) Collisional model of asteroids and their debris. J. Geophys. Res., 74, 2531-2554.

Durda D., Greenberg R., and Jedicke R. (1998) Collisional models and scaling laws: A new interpretation of the shape of the Main-Belt asteroid distribution. Icarus, 135, 431-440.

Gradie J. C., Chapman C. R., and Tedesco E. W. (1989) Distribution of taxonomic classes and the compositional structure of the Asteroid Belt. In Asteroids II (R. P. Binzel et al., eds.), pp. 316-335. Univ. of Arizona, Tucson.

Grieve R. A. F. and Shoemaker E. M. (1994) The record of the past impacts on Earth. In Hazards Due to Comets and Asteroids (T. Gehrels, ed.), pp. 417-462. Univ. of Arizona, Tucson.

Hartmann W. K. (1965) Terrestrial and lunar flux of meteorites in the last two billion years. Icarus, 4, 157-165.

Hartmann W. K. (1966) Martian cratering. Icarus, 5, 565-576.

Hartmann W. K. (1970) Lunar cratering chronology. Icarus, 13, 299-301.

Hartmann W. K. (1977) Relative crater production rates on planets. Icarus, 31, 260-276.

Hartmann W. K. (1995) Planetary cratering I: Lunar highlands and tests of hypotheses on crater populations. Meteoritics, 30, 451-467.

Hartmann W. K. (1999) Martian cratering VI. Crater count isochrons and evidence for recent volcanism from Mars Global Surveyor. Meteoritics \& Planet. Sci., 34, 167-177.

Hartmann W. K. and Neukum G. (2001) Crater chronology and the evolution of Mars. In Chronology and Evolution of Mars (R. Kallenbach et al., eds.), pp. 165-194. Kluwer, Dordrecht.
Hartmann W. K., Strom R. G., Weidenschilling S. J., Blasius K. R., Woronow A., Dence M. R., Grieve R. A. F., Diaz J., Chapman C. R., Shoemaker E. M., and Jones K. L. (1981) Chronology of planetary volcanism by comparative studies of planetary craters. In Basaltic Volcanism on the Terrestrial Planets, pp. 1050-1127. Pergamon, New York.

Hartmann W. K., Berman D., Esquerdo G. A., and McEwen A. (1999a) Recent martian volcanism: New evidence from Mars Global Surveyor (abstract). In Lunar and Planetary Science $X X X$, Abstract \#1270. Lunar and Planetary Institute, Houston (CD-ROM).

Hartmann W. K., Malin M. M., McEwen A., Carr M., Soderblom L., Thomas P., Danielson E., James P., and Veverka J. (1999b) Evidence for recent volcanism on Mars from crater counts. $\mathrm{Na}$ ture, 397, 586-589.

Hartmann W K., Farinella P., Vokrouhlický D., Weidenschilling S. J., Morbidelli A., Marzari F., Davis D. R., and Ryan E. (1999c) Reviewing the Yarkovsky effect: New light on the delivery of stone and iron meteorites from the asteroid belt. Meteoritics \& Planet. Sci., 34, 161-167.

Hartmann W. K., Ryder G., Dones L., and Grinspoon D. (2000) The time-dependent intense bombardment of the primordial Earth/moon system. In Origin of the Earth and Moon (R. M. Canup and K. Righter, eds.), pp. 493-512. Univ. of Arizona, Tucson.

Hiesinger H., Jaumann R., Neukum G., and Head J. W. III (2000) Ages of mare basalts on the lunar nearside. J. Geophys. Res., 105, 29239-29776.

Hughes D. W. (2000) A new approach to the calculation of the cratering rate of the Earth over the last $125 \pm 20$ m.y. Mon. Not. R. Astron. Soc., 317, 429-437.

Ivanov B. A. (2001) Mars/moon cratering rate ratio estimates. In Chronology and Evolution of Mars (R. Kallenbach et al., eds.), pp. 87-104. Kluwer, Dordrecht.

Ivanov B. A., Basilevsky A. T., and Neukum G. (1997) Atmospheric entry of large meteoroids: Implication to Titan. Planet. Space Sci., 45, 993-1007.

Ivanov B. A., Neukum G., and Wagner R. (1999) Impact craters, NEA, and Main Belt asteroids: Size-frequency distribution (abstract). In Lunar and Planetary Science XXX, Abstract \#1583. Lunar and Planetary Institute, Houston (CD-ROM).

Ivanov B. A., Neukum G., and Wagner R. (2001) Size-frequency distributions of planetary impact craters and asteroids. In Collisional Processes in the Solar System (H. Rickman and M. Marov, eds.), pp. 1-34. Kluwer, Dordrecht.

Ivezić Ž., Tabachnik S., Rafikov R., Lupton R. H., Quinn T., Hammergren M., Eyer L., Chu J., Armstrong J. C., Fan X., Finlator K., Geballe T. R., Gunn J. E., Hennessy G. S., Knapp G. R., Leggett S. K., Munn J. A., Pier J. R., Rockosi C. M., Schneider D. P., Strauss M. A., Yanny B., Brinkmann J., Csabai I., Hindsley R. B., Kent S., Lamb D. Q., Margon B., McKay T. A., Smith J. A., Waddel P., York D. G., and the SDSS Collaboration (2001) Solar system objects observed in the Sloan Digital Sky Survey Commissioning Data. Astron. J., 122, 2749-2784.

Jedicke R. and Metcalfe T. S. (1998) The orbital absolute magnitude distributions of Main Belt asteroids. Icarus, 131, 245260.

Jedicke R., Larsen J., and Spahr T. (2002) Observational selection effects in asteroid surveys and estimates of asteroid population sizes. In Asteroids III (W. F. Bottke Jr. et al., eds.), this volume. Univ. of Arizona, Tucson.

Laskar J. (1988) Secular evolution of the solar system over 10 
million years. Astron. Astrophys., 198, 341-362.

Love S. and Ahrens T. J. (1996) Catastrophic impacts on gravity dominated asteroids. Icarus, 124, 141-155.

McKinnon W. B., Chapman C. R., and Housen K. R. (1991) Cratering of the Uranian satellites. In Uranus (J. T. Bergstralh et al., eds.), pp. 629-692. Univ. of Arizona, Tucson.

McKinnon W. B., Zahnle K. J., Ivanov B. A., and Melosh H. J. (1997) Cratering on Venus: Models and observations. In Venus II (S. W. Bougher et al., eds.), pp. 969-1014. Univ. of Arizona, Tucson.

Melosh H. J. and Ryan E. V. (1997) Note: Asteroids shattered but not dispersed. Icarus, 129, 562-564.

Melosh H. J. and Ivanov B. A. (1999) Impact crater collapse. Annu. Rev. Earth Planet. Sci., 27, 385-415.

Morbidelli A., Bottke W. F., Jedicke R., Michel P., and Tedesco E. F. (2001) NEO albedo distribution and impact hazards (abstract). Bull. Am. Astron. Soc., 33, 1137.

Morbidelli A., Bottke W. F. Jr., Froeschlé C. H., and Michel P. (2002) Origin and evolution of near-Earth objects. In Asteroids III (W. F. Bottke Jr. et al., eds.), this volume. Univ. of Arizona, Tucson.

MPC (2001) Asteroid Observing Services. Minor Planet Center Data File available on line at http://asteroid.lowell.edu.

Nemtchinov I. V., Svetsov V. V., Kosarev I. B., Golub' A. P., Popova O. P., Shuvalov V. V., Spalding R. E., Jacobs C., and Tagliaferri E. (1997) Assessement of kinetic energy of meteoroids detected by satellite-based light sensors. Icarus, 130, 259-274.

Neukum G. (1983) Meteoritenbombardement and datierung planetarer oberfluchen. Habilitation Dissertation for Faculty Membership, University of Munich. 186 pp.

Neukum G. and Ivanov B. A. (1994) Crater size distribution and impact probabilities on Earth from lunar, terrestrial-planet, and asteroid cratering data. In Hazards Due to Comets and Asteroids (T. Gehrels, ed.), pp. 359-416. Univ. of Arizona, Tucson.

Neukum G., Ivanov B., and Hartmann W. K. (2001) Cratering records in the inner solar system. In Chronology and Evolution of Mars (R. Kallenbach et al., eds.), pp. 55-86. Kluwer, Dordrecht.

Rabinowitz D. L. (1993) The size-distribution of the Earth-approaching asteroids. Astrophys. J., 407, 412-427.

Rabinowitz D. L. (1997) Are Main-Belt asteroids a sufficient source for the Earth-approaching asteroids? Part II. Predicted vs. observed size distribution. Icarus, 130, 287-295.

Rabinowitz D. L., Bowell E., Shoemaker E., and Muinonen K. (1994) The population of Earth-crossing asteroids. In Hazards Due to Comets and Asteroids (T. Gehrels, ed.), pp. 285-312. Univ. of Arizona, Tucson.
Rabinowitz D. L., Helin E., Lawrence K., and Pravdo S. (2000) A reduced estimate of the number of kilometer-sized near-Earth asteroids. Nature, 403, 165-156.

Schmidt R. M. and Housen K. R. (1987) Some recent advances in the scaling of impact and explosion cratering. Intl. J. Impact Engng., 5, 543-560.

Sekanina Z. and Yeomans D. K. (1984) Close encounters and collisions of comets with the earth. Astron. J., 89, 154-161.

Shoemaker E. M. and Wolfe R. (1982) Cratering time scales for the Galilean satellites. In Satellites of Jupiter (D. Morrison, ed.), pp. 277-339. Univ. of Arizona, Tucson.

Stöffler D. and Ryder G. (2001) Stratigraphy and isotope ages of lunar geologic units: Chronological standard for the inner solar system. In Chronology and Evolution of Mars (R. Kallenbach et al., eds.), pp. 9-54. Kluwer, Dordrecht.

Strom R. G. and Neukum G. (1988) The cratering record on Mercury and the origin of impacting objects. In Mercury (F. Vilas et al., eds.), pp. 336-373. Univ. of Arizona, Tucson.

Strom R. G., Croft S. K., and Barlow N. G. (1992) The Martian impact cratering record. In Mars (H. H. Kieffer et al., eds.), pp. 383-423. Univ. of Arizona, Tucson.

Stuart J. S. (2001) A near-Earth asteroid population estimate from the LINEAR survey. Science, 294, 1691-1693.

van Houten C. J., van Houten-Groeneveld I., Herget P., and Gehrels T. (1970) The Palomar-Leiden survey of faint minor planets. Astron. Astrophys. Suppl., 2, 339-448.

Veverka J., Thomas P., Harch A., Clark B., Bell J. F. III, Carcich B., Joseph J., Chapman C., Merline W., Robinson M., Malin M., McFadden L. A., Murchie S., Hawkins S. E. III, Farquhar R., Izenberg N., and Cheng A. (1997) NEAR's flyby of 253 Mathilde: Images of a C asteroid. Science, 278, 2109-2114.

Veverka J., Robinson M., Thomas P., Murchie S., Bell J. F., Izenberg N., Chapman C., Harch A., Bell M., Carcich B., Cheng A., Clark B., Domingue D., Dunham D., Farquhar R., Gaffey M. J., Hawkins E., Joseph J., Kirk R., Li H., Lucey P., Malin M., Martin P., McFadden L., Merline W. J., Miller J. K., Owen W. M., Peterson C., Prockter L., Warren J., Wellnitz D., Williams B. G., and Yeomans D. K. (2000) NEAR at Eros: Imaging and spectral results. Science, 289, 2088-2097.

Weissmann P. R., Bottke W. F. Jr., and Levison H. F. (2002). Evolution of comets into asteroids. In Asteroids III (W. F. Bottke Jr. et al., eds.), this volume. Univ. of Arizona, Tucson.

Wetherill G. W. (1967) Collisions in the asteroid belt. J. Geophys. Res., 72, 2429-2444.

Wilhelms D. E., McCauley J. F., and Trask N. J. (1987) The Geologic History of the Moon. U.S. Geol. Survey Prof. Paper 1348. $302 \mathrm{pp}$. 